\title{
Le troisième récepteur de la dopamine Une nouvelle cible d'action des neuroleptiques
}

La dopamine est un important neuromédiateur cérébral impliqué dans les contrôles des fonctions motrices, émotionnelles et cognitives. L'altération du fonctionnement des systèmes dopaminergiques est à l'origine d'affections neurologiques et psychiatriques telles que la maladie de Parkinson et la Schizophrénie.

Les techniques de la biologie moléculaire ont récemment permis des avancées importantes dans la connaissance des récepteurs à la dopamine. On connaît depuis une dizaine d'années deux récepteurs, appelés $D_{1}$ et $D_{2}$, qui ont été au départ différenciés par leurs effets sur l'AMP cyclique, le premier activant la synthèse du messager intracellulaire alors que le deuxième l'inhibe. Grâce à l'homologie de séquence qui existe dans la famille des récepteurs couplés aux protéines $G$, à laquelle appartiennent les récepteurs à la dopamine, le récepteur $D_{2}$ avait été cloné en 1988 [1]. Trois publications récentes [2-4] rapportent le clonage du récepteur $D_{1}$ humain; l'ARN messager de ce récepteur est réparti dans toutes les régions dopaminergiques à l'exception de l'hypophyse et, une fois exprimé dans des cellules en culture, il présente toutes les caractéristiques pharmacologiques attendues. Son gène est localisé sur le chromosome 5 à proximité de ceux d'autres récepteurs couplés aux pro-
Les neuroleptiques, qui bloquent l'action de la dopamine, constituent le traitement d'élection des manifestations délirantes et des états d'agitation rencontrés au cours des psychoses aiguës et des formes chroniques de schizophrénie. Cependant, les propriétés bénéfiques des neuroleptiques s'accompagnent d'effets secondaires indésirables, parfois invalidants, dont les plus gênants sont d'ordre neurologique : rigidité musculaire, ralentissement moteur et après des traitements prolongés, la survenue de mouvements anormaux involontaires (dyskinésies tardives). Les effets thérapeutiques aussi bien que les effets secondaires étaient expliqués par l'interaction des neuroleptiques avec le récepteur $\mathrm{D}_{2}$, le récepteur $D_{1}$ reconnaissant beaucoup moins bien et de façon très variable ces molécules.

Certains neuroleptiques considérés comme atypiques (exemple le sulpirịde) se distinguent des neuroleptiques typiques (exemple le halopéridol) par les propriétés de leur liaison à des membranes de cerveau [5] et par leur capacité d'inhiber sélectivement des comportements stéréotypés induits chez l'animal par l'administration d'agonistes dopaminergiques [5]. De plus, l'utilisation de ces neuroleptiques atypiques en clinique s'accompagne d'effets secondaires plus discrets et certains d'entre eux améliorent les symptômes négatifs de la schizophrénie. Cette série d'observations s'accommodait mal avec l'idée communément admise que le récepteur $\mathrm{D}_{2}$ était la cible unique des neuroleptiques ; c'est ce qui nous a conduits à rechercher d'autres récepteurs dopaminergiques.

Dans un article récent [6], nous décrivons le clonage et la caractérisation d'un nouveau récepteur, nommé $\mathrm{D}_{3}$, qui se distingue des récepteurs $D_{1}$ et $D_{2}$ par sa localisation et sa pharmacologie.

Nous avons utilisé une portion du gène du récepteur $D_{2}$ pour cribler une banque d'ADNc de cerveau de rat. Ce criblage, joint à la technique de PCR, nous a permis d'isoler un clone d'ADNc, dont la protéine déduite présente une homologie de $50 \%$ avec le récepteur $\mathrm{D}_{2}$.

Un des faits remarquables tient à la localisation de ce nouveau récepteur $\mathrm{D}_{3}$. L'hybridation in situ de coupes de cerveau de rat avec des sondes nucléotidiques spécifiques du récepteur $\mathrm{D}_{2}$ ou $\mathrm{D}_{3}$ (figure 1) montre que les distributions des ARNm des deux récepteurs sont très différentes. Celle du messager du récepteur $\mathrm{D}_{2}$ est parallèle à celle des récepteurs marqués par l'iodosulpiride- ${ }^{25}$ I, qui est reconnu par les récepteurs $D_{2}$ et $\mathrm{D}_{3}$, avec de fortes densités dans l'ensemble du striatum. En revanche, la distribution de l'ARNm du récepteur $\mathrm{D}_{3}$ est restreinte à la partie ventrale du striatum, au noyau 


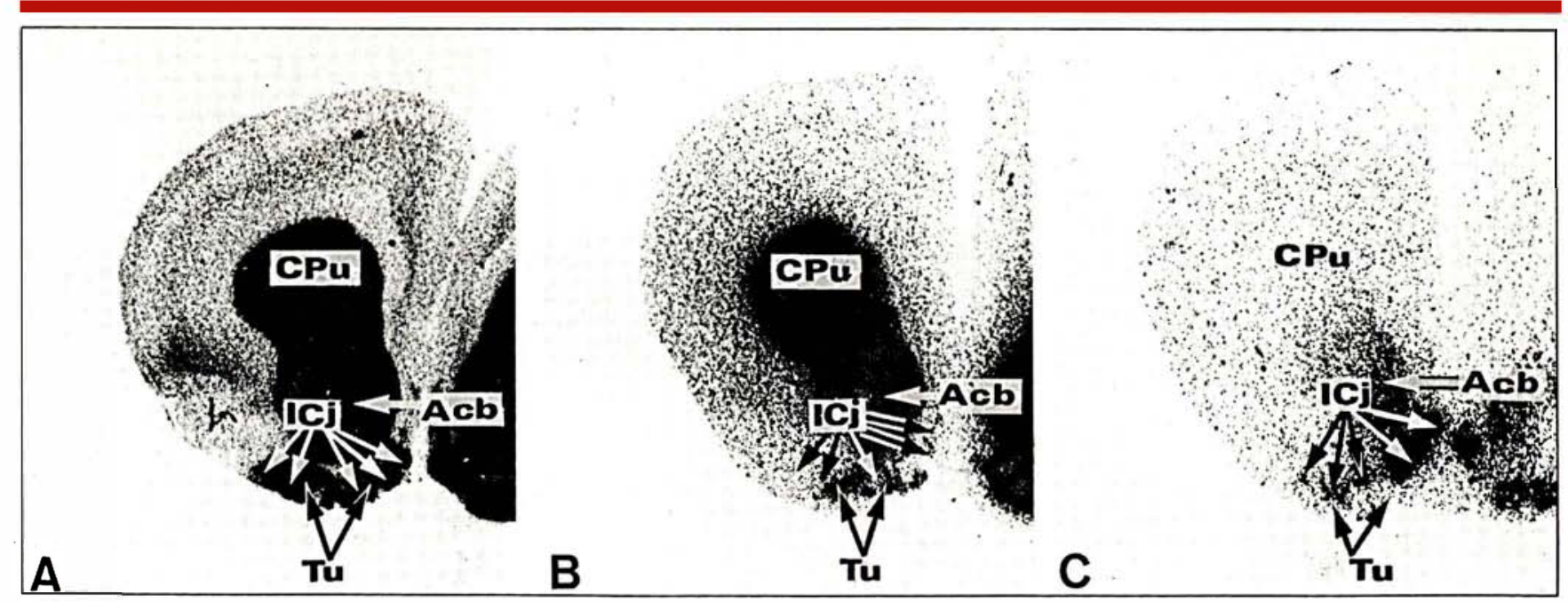

Figure 1. Localisation autoradiographique des sites de liaisons et des $A R N$ messagers des récepteurs $D_{2}$ et $D_{3}$ sur une coupe frontale de cerveau de rat. La distribution des ARNm du récepteur $D_{2}$ (B) est parallèle à celle des sites de liaison dopaminergiques $\left(D_{2}+D_{3}\right)$ marqués par un ligand radioactif (A) avec de fortes densités dans I'ensemble du striatum (CPu). La distribution des $A R N m$ du récepteur $D_{3}(C)$ est resteinte à un territoire appartenant au système limbique constitué des parties ventromédianes du striatum et du noyau accumbens (Acb), et du tubercule olfactif (Tu), particulièrement les ilots de Calleja (ICj).

\begin{tabular}{|c|c|c|c|}
\hline \multicolumn{4}{|c|}{ FICHE D'IDENTITÉ DES TROIS RÉCEPTEURS DOPAMINERGIQUES } \\
\hline & Récepteur $D_{1}$ & Récepteur $\mathrm{D}_{2}$ & Récepteur $D_{3}$ \\
\hline Localisation & $\begin{array}{l}\text { Striatum } \\
\text { Glande parathyroïde }\end{array}$ & $\begin{array}{l}\text { Striatum } \\
\text { Hypophyse }\end{array}$ & Système limbique \\
\hline Rôles & $\begin{array}{l}\text { Sécrétion } \\
\text { de I'hormone } \\
\text { parathyroïde }\end{array}$ & $\begin{array}{l}\text { Motricité } \\
\text { Sécrétions } \\
\text { hypophysaires }\end{array}$ & $\begin{array}{l}\text { Émotions } \\
\text { Processus cognitifs }\end{array}$ \\
\hline $\begin{array}{l}\text { Neuroleptiques } \\
\text { les mieux reconnus }\end{array}$ & Flupentixol & $\begin{array}{l}\text { Neuroleptiques typi- } \\
\text { ques (forts effets se- } \\
\text { condaires moteurs) } \\
\text { Ex. : halopéridol }\end{array}$ & $\begin{array}{l}\text { Neuroleptiques aty- } \\
\text { piques (moins d'ef- } \\
\text { fets secondaires } \\
\text { moteurs, plus désin- } \\
\text { hibiteurs) } \\
\text { Ex. : sulpiride }\end{array}$ \\
\hline $\begin{array}{l}\text { Identité } \\
\text { moléculaire* }\end{array}$ & $\begin{array}{l}\text { Protéines de } \\
446 \text { acides aminés }\end{array}$ & $\begin{array}{l}\text { Protéines de } \\
444 \text { acides aminés }\end{array}$ & $\begin{array}{l}\text { Protéines de } \\
446 \text { acides aminés } \\
50 \% \text { d'homologie } \\
\text { avec } D_{2} \text { l }\end{array}$ \\
\hline $\begin{array}{l}\text { Messager } \\
\text { intra-cellulaire }\end{array}$ & $\begin{array}{l}\text { gène sans intron } \\
\text { AMP cyclique }\end{array}$ & $\begin{array}{l}\text { au moins } 6 \text { introns } \\
\text { AMP cyclique }\end{array}$ & $\begin{array}{l}\text { au moins } 5 \text { introns } \\
\text { Inconnu (distinct } \\
\text { de l'AMP cyclique) }\end{array}$ \\
\hline
\end{tabular}

* Les trois récepteurs appartiennent à la famille des récepteurs liés aux G-protéines et possédant 7 fragments transmembranaires.

accumbens et aux tubercules olfactifs. Cette répartition du récepteur $\mathrm{D}_{3}$ correspond à un territoire recevant ses afférences dopaminergiques de l'aire tegmentale ventrale et ses autres afférences du cortex primitif et qui appartient au système limbique. Cela suggère que le récepteur $D_{3}$ joue un rôle important au sein du système limbique, dont on connaît depuis longtemps le rôle dans le contrôle des émotions et des processus en récepteur $\mathrm{D}_{2}$, est plus particulièrement impliqué dans le contrôle de cognitifs, alors que le striatum, riche la motricité. L'ARNm du récep- teur $\mathrm{D}_{3}$ est absent de l'hypophyse, ce qui permet de prédire que des molécules sélectives de ce récepteur n'auront pas les effets neuroendocriniens si gênants survenant lors du traitement avec certains neuroleptiques.

Le gène du récepteur $D_{3}$ a été introduit dans une cellule en culture, qui exprime alors en abondance ce récepteur, ce qui permet l'étude de sa pharmacologie. Le récepteur $\mathrm{D}_{3}$ reconnaît bien la dopamine et tous les neuroleptiques, ce qui montre qu'il constitue aussi une cible d'action pour ces molécules. Mais le fait remarquable est que les neuroleptiques typiques, comme le halopéridol, sont beaucoup moins bien reconnus par le récepteur $\mathrm{D}_{3}$ que par le récepteur $\mathrm{D}_{2}$ alors que les neuroleptiques atypiques, comme le sulpiride, sont aussi bien reconnus par les deux récepteurs. Il existe donc une relation entre le blocage efficace du récepteur $\mathrm{D}_{3}$ par les neuroleptiques atypiques et leurs propriétés cliniques particulières, caractérisées par leur faible propension à induire des effets neurologiques indésirables et leur relative efficacité à améliorer les symptômes négatifs de la schizophrénie (désintérêt, repli sur soi, autisme). Cela suggère que le blocage du récepteur $\mathrm{D}_{3}$ au niveau 
limbique est responsable des effets antipsychotiques ou, en tout cas, participe à ceux-ci alors que le blocage du récepteur $D_{2}$ au niveau striatal provoque les effets sur la motricité. Les neuroleptiques, dont le premier d'entre eux, la chlorpromazine, avait été découvert par hasard il y a quarante ans, ont souvent été sélectionnés par des tests comportementaux chez l'animal, qui ne mettent en évidence que leur action vis-à-vis du récepteur $\mathrm{D}_{2}$. C'est ainsi que sur les milliers de molécules de neuroleptiques potentiels qui ont été synthétisées, seules quelques dizaines ont été introduites en thérapeutique. La mise à la disposition de l'industrie pharmaceutique d'un test très simple d'activité anti- $\mathrm{D}_{3}$ devrait permettre dans les années qui viennent de proposer à la psychiatrie des composés nouveaux dénués d'effets secondaires et/ou à indications thérapeutiques plus adaptées.
Pierre Sokoloff, Bruno Giros, Marie-Pascale Martres, JeanCharles Schwartz

U. 109 de l'Inserm, Centre Paul-Broca, 2 ter, rue d'Alésia, 75014 Paris, France. Marie-Louise Bouthenet

Laboratoire de physiologie, faculté de pharmacie, 4, avenue de l'Observatoire, 75006 Paris, France.

\section{RÉFÉRENCES}

1. Bunzow JR, Van Tol HHM, Grandy $\mathrm{DK}$, et al. Cloning and expression of a rat $\mathrm{D}_{2}$ dopamine receptor cDNA. Nature 1988 ; 336 : 783-7.

2. Dearry A, Gingrich JA, Falardeau P, Fremeau RT, Bates MD, Caron MG'
Molecular cloning and expression of the gene for a human $\mathrm{D}_{1}$ dopamine receptor. Nature 1990 ; 347 : 72-6.

3. Zhou QZ, Grandy DK, Thambi L, et al. Cloning and expression of human and rat $D_{1}$ dopamine receptors. Nature $1990 ; 347$ : 76-80.

4. Sunahara RK, Niznik HB, Weiner DM et al. Human dopamine $D_{1}$ receptor encoded by an intronless gene on chromosome 5. Nature $1990 ; 347: 80-3$

5. Schwartz JC, Delandre M, Martres MP et al. Biochemical and behavioral identification of discriminant benzamide derivatives : new tools to differentiate subclasses of dopamine receptors. In : Catecholamines: neuropharmacology and central nervous system. Theoretical aspects. New York: Alan R. Liss Inc, 1984 : $59-72$

6. Sokoloff P, Giros B, Martres MP, Bouthenet ML, Schwartz JC. Molecular cloning and characterisation of a novel dopamine receptor $\left(D_{3}\right)$ as a target for neuroleptics. Nature 1990 ; 347 : 146-51. 